\title{
Strates
}

STRATES Matériaux pour la recherche en sciences sociales

Hors-série | 2002

Parcours dans la recherche urbaine, Michel Rochefort, un géographe engagé

\section{La formation des formateurs en Tunisie}

les études doctorales en urbanisme à l'Enau : engagement pour une nouvelle forme de coopération

\section{Mohamed El Bahi}

\section{CpenEdition}

\section{Journals}

Édition électronique

URL : http://journals.openedition.org/strates/567

DOI : $10.4000 /$ strates.567

ISSN : $1777-5442$

Éditeur

Laboratoire Ladyss

Édition imprimée

Date de publication : 1 janvier 2002

ISSN : 0768-8067

Référence électronique

Mohamed El Bahi, «La formation des formateurs en Tunisie », Strates [En ligne], Hors-série | 2002, mis en ligne le 18 mai 2005, consulté le 08 septembre 2020. URL : http://journals.openedition.org/strates/ 567 ; DOI : https://doi.org/10.4000/strates.567

Ce document a été généré automatiquement le 8 septembre 2020.

Tous droits réservés 


\title{
La formation des formateurs en Tunisie
}

\author{
les études doctorales en urbanisme à l'Enau ${ }^{1}$ : engagement pour une
} nouvelle forme de coopération

\section{Mohamed El Bahi}

1 La participation à la mise sur pied d'un projet de formation durable est une tâche qui marque pour longtemps le parcours pédagogique. Pour plusieurs générations de géographes et d'aménageurs tunisiens, Michel Rochefort est considéré, à juste titre, avec Jean Dresch et Pierre George, comme l'un des fondateurs de l'école de pensée tunisienne en matière d'organisation de l'espace.

2 Mes collègues géographes sont plus qualifiés que moi pour parler de sa contribution à la géographie humaine et urbaine en France et ailleurs. Je me limiterai donc à évoquer sa participation à l'instauration d'un enseignement de l'urbanisme à l'université de Tunis I.

3 Lorsqu'en 1990 j'ai pris la direction du nouveau département d'urbanisme de l'ITAAUT ${ }^{2}$, la mission première a été de proposer un programme d'enseignement et de choisir une équipe d'enseignants pour faire démarrer les enseignements de troisième cycle en urbanisme.

4 Les premiers collègues français ayant accepté de participer avec nous au montage de cette spécialité furent Michel Rochefort de Paris 1, Charles Goldblum et Stéphane Yérasimos de l'IFU. Au 191 rue Saint Jacques, au quatrième étage, dans son bureau du laboratoire Strates, lors de réunions courtes mais efficaces, nous fixâmes avec Michel Rochefort les programmes d'un Dess en urbanisme qui débuta en novembre 1993 et nous établîmes une liste des premiers intervenants français.

5 Ce fut le début d'une expérience qui se compléta dans un deuxième temps par le lancement d'un Dea en 1995 et l'habilitation de notre institution à dispenser des études doctorales en urbanisme. Nous nous rendîmes vite compte que les moyens mis à notre disposition n'étaient pas suffisants pour mener à bien ce programme de formation. Ainsi nous est venue l'idée d'élaborer un projet dans le cadre de la coopération franco- 
tunisienne: projet que nous avons intitulé «formation doctorale et formation des formateurs en urbanisme et aménagement urbain ».

6 Le dossier fut accepté fin 1994, ce qui nous permit de prendre en charge les frais de mission des collègues retenus pour animer des séminaires aux étudiants du Dea dont l'enseignement débuta en 1995. C'est ainsi, qu'à partir de moyens réduits, un enseignement de troisième cycle en urbanisme fut instauré à l'Enau.

7 Cette entreprise ne s'est pas effectuée sans difficultés. Ici, c'est tout un art qui a été déployé, cet art de faire passer l'idée, la bonne, sans froisser le partenaire. C'est dans une large mesure grâce à cette technique de dialogue avec autrui, sans heurter sa sensibilité, technique que maîtrise Michel Rochefort à la perfection, que nous avons pu franchir les étapes pour arriver enfin au but : créer un cadre adéquat pour former des urbanistes, scientifiquement et intellectuellement, en leur inculquant les fondements théoriques d'une discipline donnée, en l'occurence l'urbanisme, et en les sensibilisant à la portée pratique de son exercice.

8 J'ai souligné, tout au début, que Michel Rochefort avec d'autres éminents enseignants a participé à la formation de plusieurs générations de géographes tunisiens. On compte parmi ses élèves de grands responsables dans l'administration, mais aussi, ce qui est plus important, des enseignants de haut niveau engagés dans la formation de plusieurs promotions de géographes et de spécialistes de l'aménagement de l'espace. Sa participation au montage des programmes et à l'animation de plusieurs enseignements au sein du département d'urbanisme de l'Enau témoigne, encore une fois, de cet attachement que porte Michel Rochefort aux Tunisiens, loin de tout paternalisme désuet.

9 C'est dans le désintéressement qu'il conçoit la tâche de formateur, qu'il porte au niveau $\mathrm{du}$ devoir. Combien de fois l'ai-je entendu rappeler avec force que la situation de coopération dans le domaine universitaire ne doit pas être perçue en termes de profit personnel : elle doit comporter nécessairement une part d'abnégation.

10 Il y a chez Michel Rochefort un souci constant de sécuriser le partenaire. Il minimise son apport personnel à la tâche, attitude qui encourage le partenaire à construire un projet nourri des idées reçues; il se refuse à imposer un point de vue ou à flatter le pouvoir des détenteurs de la décision.

11 Ce comportement, je dirais cette philosophie, se trouve explicité dans son projet de coopération lorsqu'il considère que par la création de nouveaux axes de recherche d'intérêt commun se renforcent les échanges entre chercheurs français et tunisiens. Il s'agit d'une nouvelle forme de partenariat.

12 Je terminerai ce témoignage de reconnaissance par des considération personnelles sur l'homme, sur la rigueur de son jugement scientifique et la valeur de ses rapports humains. L'homme et son œuvre sont dignes d'intérêt, beaucoup parmi nos collègues ont dû l'évoquer amplement. Ce que je voudrais ajouter à ces témoignages portera sur une dimension de l'homme qui suscite le respect et l'attachement.

13 Les hommes de science sont souvent assimilés à des individus distants, froids, voire même inhumains. Michel Rochefort associe à l'approche rationnelle des choses le contact humain chaleureux. Dans les différentes phases de montage de nos projets communs, nous avons vécu des situations qui ont nécessité une complicité bienveillante pour nous sortir de positions conflictuelles et embarrassantes avec des collègues impatients. 
14 J'ai appris à travers cette expérience menée avec Michel Rochefort que, dans la vie universitaire, le système est articulé de telle façon que les acteurs, enseignants et chercheurs, entrent dans des actions qui les éloignent, parfois, du but premier, celui de transmettre un savoir et savoir-faire aux générations montantes. Oublier ce credo, pour lui, c'est trahir sa mission d'éducateur.

15 Cofondateur d'un enseignement de troisième cycle en urbanisme à Tunis, Michel Rochefort peut se réjouir certainement, non seulement de la portée de cet enseignement dans le cadre d'une forme de coopération avec des définitions d'avenir, mais aussi de la consolidation de cet enseignement et du prolongement de cette expérience sous la direction d'une équipe de jeunes chercheurs et enseignants à la formation desquels il a largement participé.

\section{NOTES}

1. École nationale d'architecture et d'urbanisme

2. L'Institut Technologique d'Art, d'Architecture et d'Urbanisme de Tunis.

\section{AUTEUR}

\section{MOHAMED EL BAH}

Enseignant-chercheurà l'Enau, université de Tunis I 